\title{
GENERATION OF CHIRP-FREE PICOSECOND PULSES
}

\author{
W. ZINTH, A. LAIBEREAU and W. KAISER \\ Physik Department der Technischen Universität, München, Germany
}

Received 2 May 1977

\begin{abstract}
The frequency spectrum of moderately chirped laser pulses depends upon the portion of the beam which is accepted by the spectrometer. Observation of the development of the chirp in a mode-locked pulse train allows to determine the small incipient chirp of early pulses. A product, bandwidth times pulse duration, of $0.47 \pm 0.03$ is consistently observed for single pulses switched from a passively mode-locked Nd-glass system.
\end{abstract}

It has been well established that picosecond pulses have a considerable degree of frequency drift in most experimental systems [1-3]. To determine the magnitude of the chirp, one measures independently the duration $t_{\mathrm{p}}$ and the frequency spectrum with halfwidth $\delta \nu$ of a single pulse. For coherent pulses, the product $p=\delta \nu \times t_{\mathrm{p}}$ has values of $p=0.44$ or 0.89 for gaussian or square pulses, respectively. Experimental values of $p$ as large as 100 have been observed for pulses of several picoseconds duration and some authors called their pulses "nearly bandwidth-limited", even when $p=10$.

In this letter we discuss two closely related topics. First, we consider the problem of measuring the chirp of a laser pulse correctly and, second, we present a method which allows to select an ultrafast pulse of negligible chirp. Our investigations refer to high power solid state laser systems.

The drift of the pulse frequency results from the nonlinearity of the index of refraction of the amplifying medium, glass rod or crystal, at high light intensities [4]. The chirp accumulates during the many passes of the pulse back and forth through the laser resonator. The change of the index of refraction with electric field $\delta n=n-n_{0}=n_{2}\left\langle E(t)^{2}\right\rangle$ results in a phase change

$\phi(t)=\frac{\omega_{0} z n_{2}}{c}\left\langle E(t)^{2}\right\rangle$.

The momentary frequency of the travelling light pulse $\omega_{0}+\partial \phi / \partial t$ may be calculated from the time dependence of the pulse envelope $E(t)$ [5]. The corresponding spectral intensity distribution is obtained from the relation

$E(\omega)=\frac{1}{\sqrt{2 \pi}} \int_{+\infty}^{-\infty} \mathrm{d} t E(t) \exp \left[-\mathrm{i}\left(\omega-\omega_{0}\right) t+\phi(t)\right]$.

A rough estimate of the chirp $\Delta \nu$ is given by $\Delta \nu=3 \nu_{0}$ $\delta n z / c t_{\mathrm{p}}$, where $\nu_{0}$ represents the carrier frequency, $z$ the total path length, and $\delta n \times z$ the nonlinear change of the optical path through the medium. At the frequency of a Nd-laser of $9480 \mathrm{~cm}^{-1}$, for example, one calculates $\Delta \widetilde{\nu}=3.8 \mathrm{~cm}^{-1}$, when $z=20 \mathrm{~cm}, t_{\mathrm{p}}=$ $5 \times 10^{-12} \mathrm{~s}$, and $\delta n=10^{-6}$; the latter value corresponds to phosphate glass $\left(n_{2}=1.2 \times 10^{-13} \mathrm{esu}\right)$ at a light intensity of $3 \times 10^{9} \mathrm{~W} / \mathrm{cm}^{2}$.

It is important to emphasize that the chirp is proportional to the local light intensity. One finds the largest chirp in the center of the beam and reduced values in the wings, which contain a substantial part of the total beam energy. As a result, the interpretation of the observed spectrum of the chirped pulse requires careful consideration. The measured spectrum has different shape and width depending upon the part of the beam which is accepted by the spectrometer. This point is illustrated in the following example. In fig. $1 \mathrm{a}$, the intensity distribution of a Gaussian beam is plotted versus normalized radius, where $R$ is the radius at half of the peak intensity. For an intensity level of $95 \%$, 

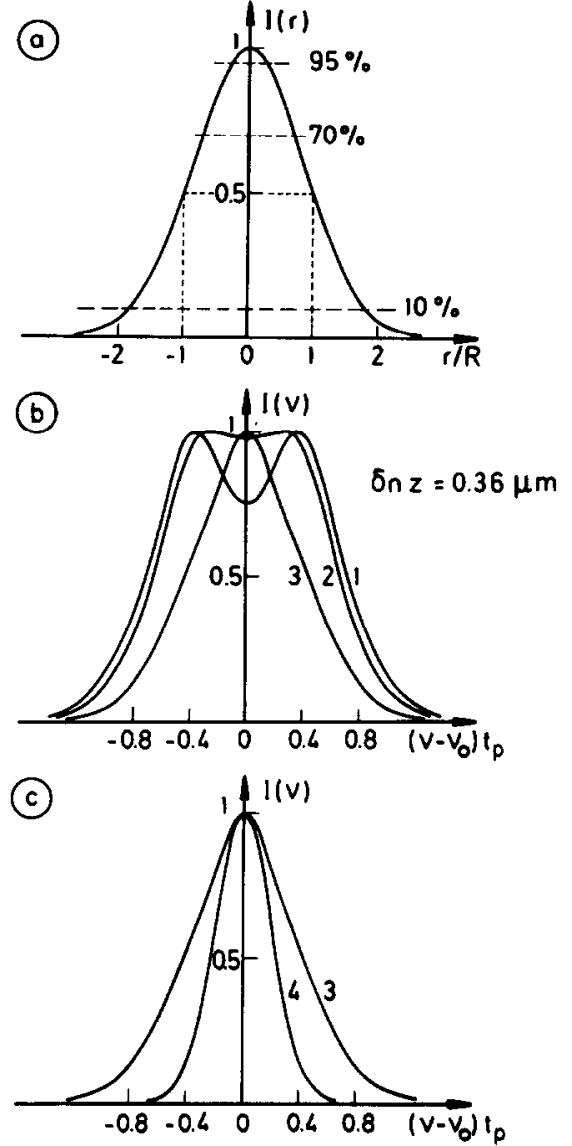

Fig. 1. (a) Gaussian intensity distribution $I(r)$ over the beam cross section versus normalized position $r / R$ (beam diameter $2 R$ ). (b) Calculated normalized spectral intensity distribution $I(\nu)$ of a gaussian pulse versus frequency in units of $t_{\mathrm{p}}^{-1}$. A nonlinear change of the optical path in the beam center of $\delta n \times z=$ $0.36 \mu \mathrm{m}$ is considered. The spectrometer is assumed to accept portions of the beam with diaphragms of radii $r=0.27 R$, $0.72 R$, and $1.82 R$, respectively, for the spectra (1), (2), and (3). (c) The spectrum $I(\nu)$ of a chirp-free pulse (4) of width $\delta \nu_{0}=0.44 / t_{\mathrm{p}}$ is compared with spectrum (3) of (b).

$70 \%$, and $10 \%$ of the peak intensity, one readily calculates beam radii of $r=0.27 R, 0.72 R$, and $1.82 R$, respectively. The light energy going through diaphragms of corresponding diameters is $5 \%, 30 \%$, and $90 \%$ of the total beam energy. In fig. 1b, three spectra (normalized to the same height) are presented. They are calculated for a nonlinear change of the optical path of $\delta n \times z=0.36 \mu \mathrm{m}$ at the peak intensity in the center of the beam. The pulse frequency is assumed to be $\widetilde{\nu}_{0}=18960 \mathrm{~cm}^{-1}$, the second harmonic of our
Nd-laser. The spectra of fig. $1 \mathrm{~b}$ correspond to three cases. The spectrometer sees (1) the center part of the beam with $r=0.27 R$ and (2), (3) two larger beam cross sections with $r=0.72 R$ and $r=1.82 R$, respectively. Case 1 gives a broad, modulated spectrum resulting from the relatively large contribution of the chirped radiation. Spectrum (3), on the other hand, shows a completely different spectral distribution, a bell-shaped form, reminiscent of a chirp-free pulse. In fig. 1c, the spectrum (3) is redrawn and compared with a truly chirp-free Gaussian spectrum of halfwidth $\Delta \nu_{0}=0.44 / t_{\mathrm{p}}$. The broadening of spectrum (3) is now apparent. The spectra of figs. $1 \mathrm{~b}$ and $\mathrm{c}$ indicate that the presence of a moderate chirp hardly can be inferred from the form of the spectrum when most of beam energy is imaged onto the spectrometer.

The results on this point may be viewed as follows: For a quantitative determination of the chirp of a light pulse, one has to know the frequency bandwidth, the time duration and the temporal shape of the pulse. The interpretation of the measured bandwidth requires the knowledge of the observed portion of the beam. Considering the difficulty of determining accurately the pulse duration and pulse shape, one recognizes the problem of establishing the degree of chirp of picosecond laser pulses.

We now discuss an experimental technique which allows us to generate single picosecond pulses of a high degree of monochromaticity. The experimental set-up [6] consists of a passively mode-locked Nd-glass laser which produces highly reproducible trains of picosecond pulses. The subsequent electro-optic switch cuts a single pulse of prescribed peak intensity from the leading part of the pulse train. The single pulse is amplified in a second glass rod with negligible additional chirp and is frequency doubled in a KDP crystal before the spectrum is analyzed by a $2 \mathrm{~m}$ grating spectrometer in conjunction with an optical multichannel analyses. Care was taken to accept the center part of the light beam with $r=0.72 R$. In fig. 2a we present part of a typical pulse train as it appears on a transient digitizer (timc resolution $0.7 \mathrm{~ns}$ ). The spectra in fig. $2 \mathrm{~b}$ correspond to the five numbered pulses. The experimentally obtained spectra show very clearly a narrow pulse (1) and the chirp of the laser pulses (3), (4), and (5). Comparison with calculated spectra suggests that spectrum (4) corresponds to a value $\delta n \times z=$ $0.36 \mu \mathrm{m}$ (see fig. $1 \mathrm{~b}$ ). Since the peak intensity of the 

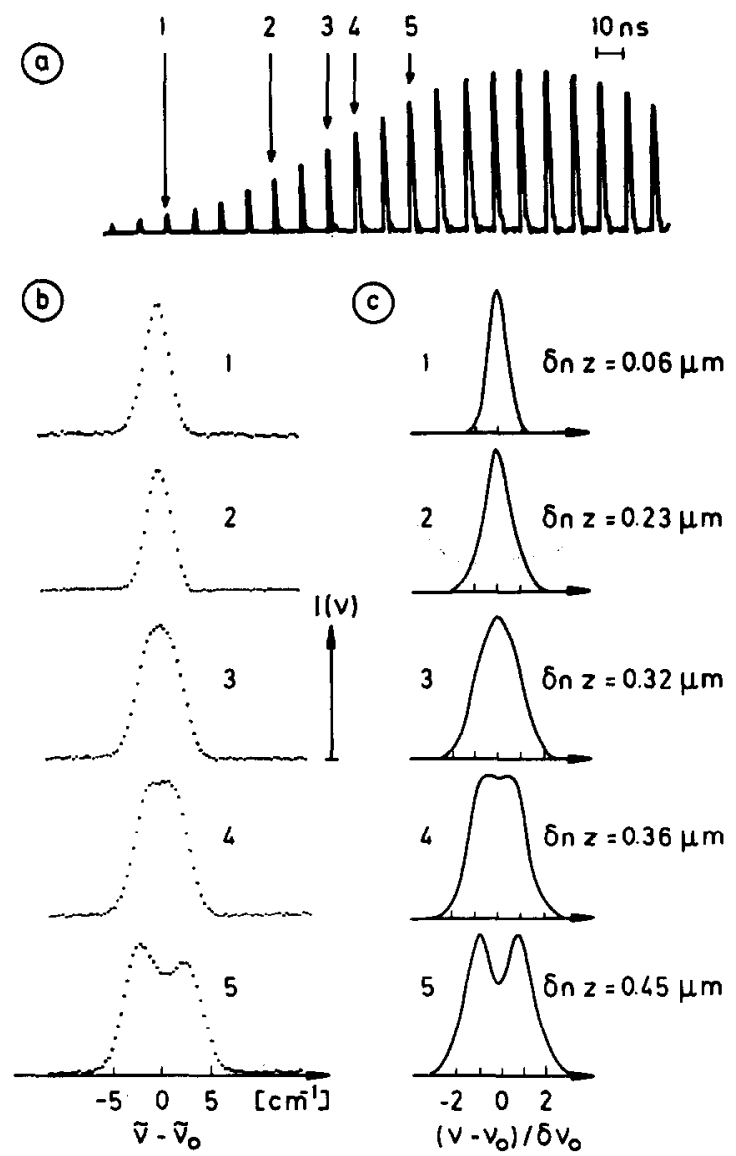

Fig. 2. (a) Rising part of the pulse train of a mode-locked Nd: glass laser as detected by a fast photodiode and a transient digitizer. (b) Observed spectra of five picosecond pulses selected from defined positions (1) to (5) of the pulse train (see (a)). Data are taken with a diaphragm of radius $r=0.72 R$. (c) The corresponding calculated spectral intensity distributions versus normalized frequency.

Comparison of (b) and (c) allows to estimate the incipient chirp of pulse (1) to be $\Delta \nu / \delta \nu_{0}=2 \times 10^{-2}$.

laser pulse is proportional to the peak height of the pulses at the digitizer (at the beginning of the pulse train) we are able to calculate $\delta n$ and the spectra of pulses (1) to (5). The computed spectra of fig. $2 \mathrm{c} \mathrm{com-}$ pare favorably with the experimental results of fig. $2 \mathrm{~b}$. Of special interest is the fact, that we are now in the position to extrapolate to earlier pulses, for instance to the pulse with number one, and make an accurate statement as to its incipient chirp. This procedure tells us that for pulse (1) we have $\Delta \nu / \delta \nu_{0} \approx 2 \times 10^{-2}$, indeed a negligible chirp of this early pulse.

We note that the preceding treatment is correct for the beginning of the pulse train where the gain in the oscillator is constant. We have made a detailed study of the chirp in our oscillator, taking into account the linear losses, the gain, the length of the amplifying medium, and the nonlinear coefficient $n_{2}$. The chirp could be predicted during the rising part of the pulse train in agreement with experimental observations. It is emphasized that the careful observation of the growth of the chirp within the pulse train and the welldefined selection of a single pulse from the early part of the train allows to generate practically chirp-free pulses.

In order to ascertain the high quality of our pulses we simultaneously measured the spectrum and the twophoton fluorescence track [7] with the help of two optical multichannel analysers (see figs. $3 \mathrm{a}$ and $\mathrm{b}$ ). We found a contrast ration of $2.9 \pm 0.2$ of the second order autocorrelation pattern suggesting good coherence of the pulses. The pulse duration was found to be $5.0 \pm 0.3$ ps. The product $p=\delta \nu \times t_{\mathrm{p}}$ was measured to be $0.47 \pm 0.03$ as an average value of 25 pulses ${ }^{\ddagger}$. To the best of our knowledge, such a small value of $p$ has not been reported previously for pulses of several picoseconds duration. The result presented here is consistent with a gaussian pulse shape ${ }^{\neq}$and gives convincing evidence that we have generated truly co-

* The statistical error (twice the standard deviation) is indicated.

₹ An approximately Gaussian pulse shape has been measured in previous investigations, see ref. [8].
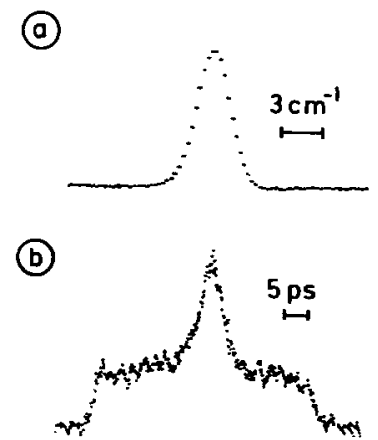

Fig. 3. Frequency width (a) and duration (b) of a single pulse selected from an early position (1) of the pulse train after conversion to the second harmonic frequency. (a) Spcctral intensity distribution as measured with a grating spectrometer and an optical multichannel analyser. (b) Second order autocorrelation pattern of the same pulse as in (a) observed by the two-photon fluorescence technique using a multichannel analyser; $t_{\mathrm{p}}=5 \mathrm{ps}, \delta \nu t_{\mathrm{p}}=0.47$. 
herent optical signals of picosecond duration.

Using the pulses described here and taking advantage of the discussion given above, we have devised a simple system to measure the nonlinear coefficient $n_{2}$ of various materials with a single picosecond pulse. Two pulses are first generated with the help of a beam splitter. One pulse is imaged at the upper part of the slit of a spectrometer while the second pulse traverses the medium of interest and is subsequently imaged at the lower part of the slit. With a twodimensional optical multichannel analyser, the two spectra are stored and slight changes of the spectra are readily detected. We are able to observe additional chirps of the second pulse of $\Delta \nu / \delta \nu \approx 0.2$. This method appears to be competitive with other techniques to measure $n_{2}$ in liquids and solids [9-11].

In closing this note we point to the importance of chirp-free pulses for coherent experiments in the picosecond time domain. In addition, there are substantially higher conversion efficiencies in the generation of new frequencies via nonlinear optical processes when working with coherent laser pulses [12].

\section{References}

[1] E.B. Tracy, Appl. Phys. Lett. 14 (1969) 112.

[2] M.A. Duguay, J.W. Hansen and S.L. Shapiro, IEEE J. Quant. Electr. QE-6 (1790) 725;
V.V. Korobkin, A.A. Malyutin and A.M. Prokhorov, JETP Lett. 12 (1970) 150;

D.J. Bradley, G.H.C. New and S.J. Caughey, Phys. Lett. 32 A (1970) 313.

[3] R.C. Eckardt, C.H. Lee and J.N. Bradford, Appl. Phys. Lett. 19 (1971) 420;

D. von der Linde, IEEE J. Quant. Electr. QE-8 (1972) 328;

M.C. Richardson, IEEE J. Quant. Electr. QE-9 (1973) 768.

[4] R.C. Eckardt, C.H. Lee, J.N. Bradford, Opto-electronics 6 (1974) 67.

[5] A. Laubereau, Phys. Lett. 29A (1969) 539;

A. Laubereau and D. von der Linde, Z. Naturf. 25a (1970) 1626.

[6] A. Laubereau, W. Kaiser, Opto-electronics 6 (1974) 1.

[7] J.A. Girdmaine, P.M. Rentzepis, S.J. Shapiro and K.W. Wecht, Appl. Phys. Lett. 11 (1967) 216.

[8] D. von der Linde, A. Laubereau and W. Kaiser, Phys, Rev. Leti. 26 (1971) 954.

[9] G. Mayer and F. Gives, C.R. Acad. Sci. 258 (1964) 2039.

[10] A. Feldman, D. Horowitz and R.M. Waxler, IEEE J. Quant. Electr. QE-9 (1973) 1054;

W.L. Smith, J.H. Bechtel and N. Bloembergen, Phys. Rev. B12 (1975) 706;

A. Owyoung, IEEE J. Quant. Electr. QE-9 (1973) 1064; M.D. Levenson, IEEE J. Quant. Electr. QE-10 (1974) 110.

[11] E.S. Bliss, D.R. Speck and W.W. Simmons, Appl. Phys. Lett. 25 (1974) 728;

M.J. Moran, C.-Y. She, and R.L. Carman, IEEE J. Quant. Electr. QE-11 (1975) 259.

[12] Yu.N. Karamzin and A.P. Sukhorukov, Sov. J. Quant. Electron. 5 (1975) 496. 